\title{
Programa de muestreo para el pulgón amarillo del sorgo, Melanaphis sacchari (Zehntner)
}

\section{Sampling program for the sugarcane aphid, Melanaphis sacchari (Zehntner)}

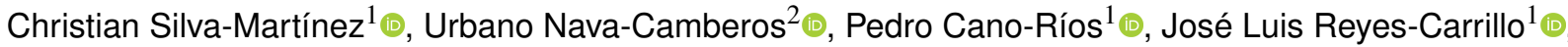 \\ Verónica Ávila-Rodríguez² (D, José Luis García-Hernández ${ }^{3 *}$ \\ ${ }^{1}$ Universidad Autónoma Agraria Antonio Narro-Unidad Laguna. Periférico Raúl López Sánchez S/N, Colonia Valle Verde, CP. 27054. \\ Torreón, Coahuila, México. \\ ${ }^{2}$ Facultad de Ciencias Biológicas-Universidad Juárez del Estado de Durango. Av. Universidad S/N, Fracc. Filadelfia, CP. 35010 . Gómez \\ Palacio, Durango, México. \\ ${ }^{3}$ Facultad de Agricultura y Zootecnia-Universidad Juárez del Estado de Durango. Carretera Gómez Palacio - Tlahualilo Km. 32. Ej. \\ Venecia, Gómez Palacio, Durango. México. \\ *Autor de correspondencia: luis_garher@hotmail.com
}

Nota científica recibida: 19 de noviembre de 2018 aceptada: 05 de mayo de 2019

RESUMEN. El presente estudio se realizó con el objetivo de desarrollar un programa de muestreo para el pulgón amarillo del sorgo (Melanaphis sacchari Zehntner). Esta plaga es actualmente la de mayor importancia económica en el cultivo de sorgo en México y otros países. Se efectuaron cinco muestreos de la plaga en 10 predios de sorgo de la Comarca Lagunera. Se determinó la relación varianza $\left(\mathrm{S}^{2}\right)$-media $(\mathrm{m})$ para el número de pulgones mediante el modelo de la Ley de Poder de Taylor y se estimaron los tamaños de muestra óptimos. Las densidades máximas variaron de 394.7 a 754.1 pulgones por hoja en el mes de julio. A través del modelo de regresión obtenido $\left[\log \left(S^{2}\right)=0.4374+1.6513 \log (\mathrm{m}), \mathrm{r}^{2}=0.98\right]$ se determinó que para el muestreo del pulgón amarillo del sorgo se deben inspeccionar al menos 39 hojas por predio, con una precisión del $80 \%$.

Palabras clave: Densidad, hojas infestadas, importancia económica, inspeccionar, promedios máximos.

ABSTRACT. The present study was conducted in order to develop a sampling program for the sugarcane aphid, Melanaphis sacchari Zehntner. This pest is currently of the greatest economic importance in the cultivation of sorghum in Mexico and other countries. There were five samplings of the insect pest in 10 sorghum plots from the Comarca Lagunera. The variance $\left(\mathrm{S}^{2}\right)$ - mean $(\mathrm{m})$ relationship for the number of sugarcane aphids was determined using the Taylor's Power Law model and the optimal sample sizes were estimated. Maximum densities ranged from 394.7 to 754.1 sugarcane aphids per leaf in the month of July. Through the obtained regression model $\left[\log \left(S^{2}\right)=0.4374+1.6513 \log (m), r^{2}=0.98\right]$ it was determined that for sampling the sugarcane aphid at least 39 sorghum leaves must be inspected per plot, with an accuracy of $80 \%$.

Key words: Density, infested leaves, economic importance, inspect, maximum averages.

\section{INTRODUCCIÓN}

El Pulgón Amarillo del Sorgo (PAS), Melanaphis sacchari Zehntner (Hemiptera: Aphididae) es una especie invasiva de reciente introducción en México (Peña-Martínez et al. 2015). Se ha convertido de forma rápida en una plaga de importancia en el cultivo del sorgo en varios estados (TejedaReyes et al. 2017). En México, se tienen reportes de la presencia de la plaga en todas las regiones productoras de sorgo (Villanueva et al. 2014), en las que la plaga se comporta de forma agresiva y capaz de desplazarse de forma rápida causando daños severos tanto en sorgo de grano como forrajero (SENASICA 2014, 2017, Rodríguez-del-Bosque y Terán 2015). El daño que ocasiona al sorgo el pulgón amarillo depende de varios factores, entre los que se incluyen la densidad de áfidos y la duración de la infestación, siendo la temperatura la variable meteorológica de mayor efecto en la tasa de desarrollo del PAS (Singh et al. 2004). De acuerdo con Setokuchi (1988) a $15{ }^{\circ} \mathrm{C}$ tarda 10.9 días, a 20 
${ }^{\circ} \mathrm{C} 7.3$ días, a $25{ }^{\circ} \mathrm{C} 5.2$ días y a $30{ }^{\circ} \mathrm{C} 3.5$ días para reproducirse, con óptimo entre $20^{\circ}$ y $25^{\circ} \mathrm{C}$.

Los daños incluyen la decoloración marrón de la hoja, seguido de la clorosis, necrosis, retraso del crecimiento y retraso en la floración, llenado de grano deficiente, lo que ocasiona pérdidas significativas de calidad y rendimiento. EI PAS se alimenta de la superficie abaxial de las hojas, las infestadas se cubren a menudo con fumagina que crece en la mielecilla producida por el PAS, lo que afecta la capacidad fotosintética de la planta (Villanueva et al. 2014). Se tienen informes de que el PAS puede transmitir el virus Sugarcane mosaci virus (SCMV), Sugarcane yellow leaf virus (SCYLV) y otras enfermedades (Schenk y Lehrer 2000, White et al. 2001).

Los estudios de dinámica de poblaciones y de umbrales económicos del PAS en sorgo grano, indican que el umbral es de 50 a 125 pulgones por hoja. Aun así, este número es relativo y depende del costo del control y del precio del grano (da Silva et al. 2014, Bowling et al. 2016). Un componente importante en los programas de manejo integrado de plagas es contar con métodos confiables y eficientes para monitorear la densidad de sus poblaciones y así poder tomar decisiones acertadas en el manejo de la población. Un programa eficiente de muestreo debería tener las siguientes características: 1) ser fácil de usar, 2) requerir un mínimo de esfuerzo y costo y 3) proporcionar estimaciones precisas de la abundancia de la plaga. El muestreo se considera un componente fundamental de las actividades de investigación en ecología poblacional, dinámica de poblaciones y el desarrollo de métodos de control alternativos (Domínguez 2011). Parte del trabajo que se tiene que hacer para lograr un programa de muestreo de plagas eficiente es definir la relación varianzamedia con respecto a una población de plagas. Al respecto, Barrera (2012) señala la importancia de la aplicación de la Ley de Poder de Taylor, para mejorar el muestreo de insectos de importancia económica. El desarrollo de un programa de control biológico involucra el conocimiento del ciclo de vida, la dispersión de la población y la densidad de la plaga. Con esos parámetros se pueden formar criterios al momento de tomar decisiones oportunas sobre actividades de manejo (Legg y Moon 1994). Debido a que no se tiene una metodología definida de muestreo ni un umbral preciso para el sorgo forrajero en el norte de México, el objetivo fue obtener datos del comportamiento y dinámica poblacional para desarrollar una metodología de muestreo del pulgón amarillo del sorgo.

\section{MATERIALES Y MÉTODOS}

\section{Ubicación y manejo de los sitios de muestreo}

El presente estudio se realizó en La Comarca Lagunera, que comprende cinco municipios del estado de Coahuila (Matamoros, San Pedro, Torreón, Viesca y Francisco I. Madero) y 10 municipios del estado de Durango (Lerdo, Gómez Palacio, Mapimí, Nazas, Rodeo, Tlahualilo, Simón Bolivar, San Juan de Guadalupe, San Luis del Cordero y San Pedro del Gallo). Se tomaron muestras de cinco predios de Durango (Pamplona, municipio de Tlahualilo; Venecia, 6 de Octubre y El Vergel, municipio de Gómez Palacio y Carlos Real, municipio de Lerdo) y cinco predios de Coahuila (ejidos Matamoros1 y Matamoros 2, municipio de Matamoros; ejidos Hormiguero y Guadalupe, municipio de Francisco I. Madero y ejido San Pedro, municipio de San Pedro). La distribución de los predios consideró una amplitud significativa en características edafoclimáticas.

El manejo agronómico en todos los sitios fue similar con respecto a la preparación del suelo, densidad y época de siembra, así como el uso de fertilizantes. Las diferencias principales fueron en el número de aplicaciones de insecticidas. De los 10 sitios de muestreo, solamente en dos (Matamoros 1 y Matamoros 2) se realizó aplicación de insecticidas convencionales. En todos los demás el control de plagas fue con los enemigos naturales presentes en cada localidad.

\section{Metodología del muestreo}

En cada predio se inspeccionaron 50 plantas tomadas al azar. Los muestreos se realizaron tomando una hoja de la parte superior de cada una de las plantas, de cada hoja se registró el número de pulgones. Se realizaron cinco muestreos, con fre- 
cuencia mensual, iniciando el 7 de abril y terminando el 3 de agosto del 2015.

\section{Determinación de la relación varianza-media (RVM) del pulgón amarillo del sorgo}

Para desarrollar el programa de muestreo para el pulgón amarillo del sorgo, primero se debe tener una muestra amplia de predios y determinar la RVM de la plaga. La metodología más aceptada para lograr esto es el modelo de la Ley del Poder de Taylor (Taylor 1961). La Ley de Poder de Taylor establece que la varianza $\left(\mathrm{s}^{2}\right)$ es proporcional a una fracción exponencial de la media de la población (m), y se calcula con la fórmula: $s^{2}=a m^{b}$, siendo los parámetros a y b estimados mediante mediante regresión lineal simple del modelo anterior. De esta manera la ecuación lineal es la siguiente:

$$
\log \left(s^{2}\right)=\log (a)+b \log (m)
$$

Donde: $s^{2}$ es la varianza del número de pulgones amarillos por hoja, $\mathrm{m}$ es el promedio del número de pulgones amarillos por hoja, el parámetro b es la pendiente o coeficiente de regresión, este es un índice de la agregación del insecto, de modo que si $b<1$ la disposición del insecto es regular, si $b=1$ la disposición es al azar y si b > 1 la disposición es agregada; mientras que el parámetro a, el intercepto, es un factor de escala relacionado al tamaño de la unidad de muestreo (Taylor 1961).

\section{Estimación del tamaño de muestra óptimo para el pulgón amarillo del sorgo}

Para estimar el tamaño de muestra óptimo (n) se empleó la fórmula: $n=\left(a m^{b-2}\right) l c^{2}$. Donde: $c$ es la precisión requerida como una proporción de la media, si c $=0.2$, el error estándar equivale al $20 \%$ de la media y la precisión es del $80 \%$ (Taylor 1961). Los niveles de precisión usados fueron 0.10 y 0.20 , de acuerdo con Naranjo y Flint (1995), quienes sugieren valores de $c$ entre 0.1 y 0.25

\section{RESULTADOS Y DISCUSIÓN}

\section{Densidades y niveles de Infestación del pulgón amarillo del sorgo}

La Tabla 1 muestra los datos obtenidos durante los muestreos del PAS en el ciclo agrícola primaveraverano en los predios bajo estudio. La densidad poblacional del PAS en el cultivo del sorgo puede presentar variaciones, como resultado de la influencia de labores culturales, control químico y las condiciones climáticas. Las infestaciones del PAS en el cultivo de sorgo forrajero fueron bajas de abril a mayo del 2015 ( $\leq 2.0$ PAS por hoja), pero posteriormente se incrementó de forma exponencial al final del ciclo del cultivo. Las densidades máximas de la plaga fueron de 394.7 a 754.1 PAS por hoja en el mes de julio. Estas densidades del PAS rebasan los umbrales económicos de 50 a 125 PAS por hoja determinados en sorgo para grano (da Silva et al. 2014 y Bowling et al. 2016). En el caso del sorgo forrajero las densidades del PAS que causan pérdidas de rendimiento y económicas; así como los umbrales económicos correspondientes pueden ser mayores a los de sorgo para grano. Al respecto Tejeda-Reyes et al. (2017) mencionan promedios máximos del PAS por hoja de 353.8, mediante muestreo directo a 30 hojas de sorgo por predio en el estado de Guerrero. Mientras que Vázquez-Navarro et al. (2016) coincide con lo observado en el presente estudio reportando que en el mes de julio se presenta mayor densidad poblacional del PAS.

El áfido M. sacchari pueden alcanzar los 30 000 individuos por planta, lo que provoca daños por la succión de la savia de la hoja. Las plantas infestadas se tornan rojizas por las lesiones, lo cual ocasiona cambios fisiológicos como, clorosis, marchitamiento de la hoja, disminuye la asimilación de nutrientes, lo que provoca retraso en el crecimiento y por lo tanto disminución del rendimiento del cultivo (SENASICA 2014). El daño es menor cuando las poblaciones son bajas, el problema consiste en que su capacidad de reproducción es dos veces más alta comparada con las especies de áfidos que atacan comúnmente al sorgo; por lo que $M$. sacchari se desarrolla de forma adecuada cuando la temperatura se encuentra por 
Tabla 1. Promedios, varianzas y porcentajes de hojas infestadas por el pulgón amarillo del sorgo, Melanaphis sacchari, en sorgo forrajero.

\begin{tabular}{|c|c|c|c|c|c|c|}
\hline \multirow[t]{2}{*}{ Predio } & \multirow{2}{*}{ Estadístico } & \multicolumn{5}{|c|}{ Fecha de muestreo } \\
\hline & & 7 abril & 15 mayo & 15 junio & 11 julio & 1 agosto \\
\hline \multirow[t]{3}{*}{1} & Media & 0.3 & 1.6 & 0.7 & 552.2 & 4.7 \\
\hline & Varianza & 1.4 & 5.9 & 1.7 & 117153.3 & 58.0 \\
\hline & $\%$ hojas inf. & 10.0 & 36.0 & 28.0 & 100.0 & 38.0 \\
\hline \multirow[t]{3}{*}{2} & Media & 0.5 & 1.4 & 63.7 & 719.2 & 7.0 \\
\hline & Varianza & 1.9 & 4.5 & 807.1 & 291006.9 & 96.5 \\
\hline & $\%$ hojas inf. & 20.0 & 36.0 & 100.0 & 100.0 & 44.0 \\
\hline \multirow[t]{3}{*}{3} & Media & $-^{a}$ & 1.7 & 25.4 & 394.7 & 511.0 \\
\hline & Varianza & - & 5.5 & 556.7 & 104492.9 & 148321.0 \\
\hline & $\%$ hojas inf. & - & 40.0 & 96.0 & 100.0 & 100.0 \\
\hline \multirow[t]{3}{*}{4} & Media & - & 1.7 & 51.0 & 754.1 & 6.8 \\
\hline & Varianza & - & 6.2 & 1406.0 & 360291.2 & 86.6 \\
\hline & $\%$ hojas inf. & - & 38.0 & 98.0 & 100.0 & 42.0 \\
\hline \multirow[t]{3}{*}{5} & Media & - & 1.7 & 44.1 & 638.7 & 7.3 \\
\hline & Varianza & - & 5.1 & 593.4 & 459191.8 & 87.7 \\
\hline & $\%$ hojas inf. & - & 42.0 & 100.0 & 100.0 & 44.0 \\
\hline \multirow[t]{3}{*}{6} & Media & 0.3 & 1.9 & 78.3 & 551.5 & 7.6 \\
\hline & Varianza & 0.6 & 8.3 & 3213.6 & 117464.7 & 104.8 \\
\hline & $\%$ hojas inf. & 18.0 & 34.0 & 100.0 & 100.0 & 44.0 \\
\hline \multirow[t]{3}{*}{7} & Media & - & 1.0 & 42.6 & 553.8 & 8.9 \\
\hline & Varianza & - & 2.9 & 237.7 & 110102.5 & 103.3 \\
\hline & $\%$ hojas inf. & - & 28.0 & 100.0 & 100.0 & 52.0 \\
\hline \multirow[t]{3}{*}{8} & Media & - & 1.5 & 49.0 & 573.0 & 8.2 \\
\hline & Varianza & - & 4.8 & 321.5 & 155078.9 & 115.1 \\
\hline & $\%$ hojas inf. & - & 34.0 & 100.0 & 100.0 & 46.0 \\
\hline \multirow[t]{3}{*}{9} & Media & - & 1.8 & 39.7 & 444.6 & 9.2 \\
\hline & Varianza & - & 6.3 & 373.7 & 45982.0 & 118.3 \\
\hline & $\%$ hojas inf. & - & 38.0 & 98.0 & 100.0 & 50.0 \\
\hline \multirow[t]{3}{*}{10} & Media & - & 1.8 & 39.8 & 618.3 & 722.4 \\
\hline & Varianza & - & 4.8 & 367.8 & 98887.7 & 130144.6 \\
\hline & $\%$ hojas inf. & - & 50.0 & 92.0 & 100.0 & 100.0 \\
\hline
\end{tabular}

arriba de los $25^{\circ} \mathrm{C}$ (Colares et al. 2015).

Las medias y las varianzas de la población del PAS se ajustaron a la ecuación, esta relación entre el promedio y la varianza de pulgones amarillos por hoja para todas las fechas de muestreo se muestra en la Figura 1.

\section{Tamaños de muestra óptimos}

Los tamaños de muestra para estimar la densidad media del PAS variaron en función de los niveles poblacionales de la plaga y niveles de precisión. Al disminuir la población media y al incrementarse la precisión ( $c=0.10$, precisión del $90 \%$ ), el tamaño de muestra fue muy alto, alrededor de 155 hojas de sorgo forrajero a muestrear. Por el contrario, se requieren tamaños de muestra bajos, 39 hojas o menos, para densidades de cinco o menos pulgones por hoja para una precisión del $80 \%(c=0.20)$
(Figura 2). Para seleccionar el tamaño de muestra apropiado se tienen las siguientes dos opciones que se pueden utilizar de forma indistinta: 1) seleccionar el tamaño de muestra más alto que funcione para densidades bajas, un pulgón en promedio por hoja, y altas, 100 ó más pulgones en promedio por hoja, del PAS. Un tamaño de muestra de 39 hojas de sorgo forrajero funciona de forma adecuada tanto en densidades bajas como altas del PAS con precisión del $80 \%$. En el muestreo preliminar se efectúa un número reducido de observaciones para obtener el promedio de insectos por hoja. Por ejemplo, considérese que se lleva a cabo un muestreo preliminar en el cultivo del sorgo forrajero y se cuentan los individuos del pulgón amarillo del sorgo en 10 hojas tomando las mismas plantas al azar en el predio y se obtiene un promedio de cinco pulgones por hoja, entonces el tamaño de muestra definitivo será de 22 hojas 


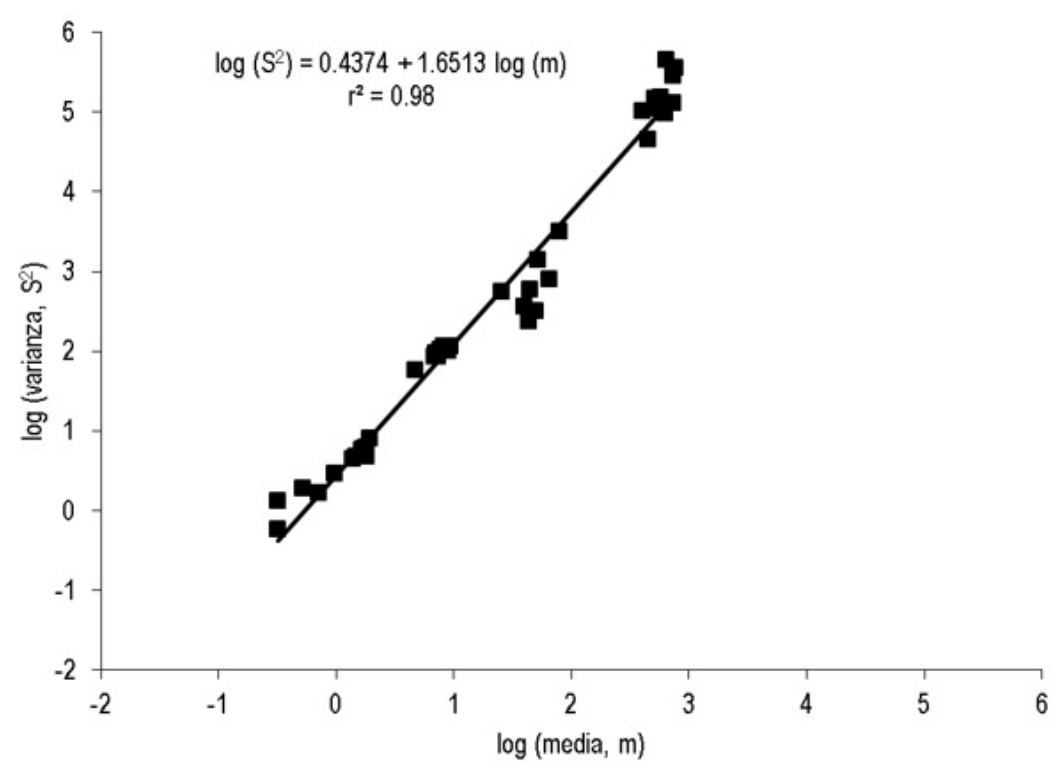

Figura 1. Relación lineal varianza-media para individuos del pulgón amarillo del sorgo Melanaphis sacchari, en hojas de sorgo forrajero mediante la Ley de Poder de Taylor.

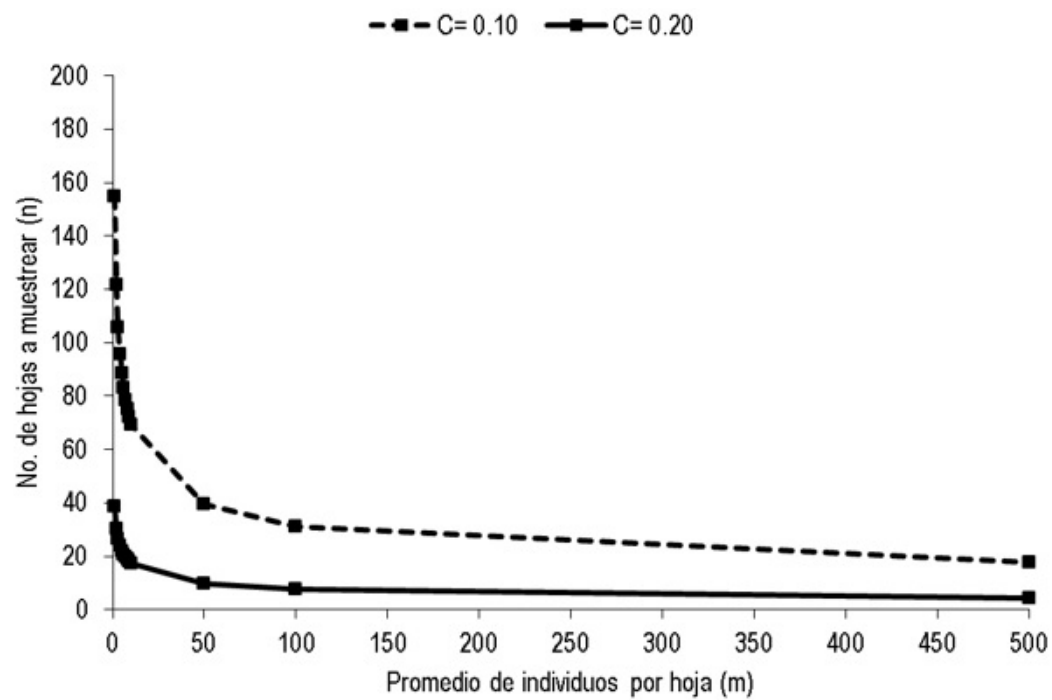

Figura 2. Relación entre el tamaño óptimo de muestra y el promedio de individuos del pulgón amarillo del sorgo Melanaphis sacchari, en hojas de sorgo forrajero para dos niveles de precisión $(\mathrm{C}=0.10$ y $\mathrm{C}=0.20)$.

para una precisión del $80 \%$ o de 88 hojas para una precisión del 90\% (Figura 2). Al respecto QuijanoCarranza et al. (2017) en el estado de Guanajuato propone el muestreo directo de 40 hojas de sorgo, de 20 plantas seleccionadas al azar; de cada planta se inspecciona una hoja de la parte superior y otra de la parte inferior para contabilizar los PAS. Pero
Norm et al. (2017) mencionan que no existen diferencias significativas en las densidades observadas de la plaga en la parte inferior y superior de la planta. De acuerdo con la metodología empleada y con los resultados obtenidos se puede concluir que las densidades máximas variaron de 394.7 a 754.1 PAS por hoja en el mes de julio. A través del modelo de regre- 
sión obtenido $\left[\log \left(S^{2}\right)=0.4374+1.6513 \log (m), r^{2}\right.$ $=0.98$ ] se determinó que para el muestreo del pulgón amarillo del sorgo se deben inspeccionar al menos 39 hojas por predio, con una precisión del $80 \%$.

\section{LITERATURA CITADA}

Barrera JF (2012) Aplicación de la Ley de Poder de Taylor al muestreo de insectos. In: Toledo J, Infante F (eds.) Manejo Integrado de Plagas. Editorial Trillas. México. pp. 47-79

Bowling RD, Brewer MJ, Kerns DL, Gordy J, Seiter N, Elliott NE, Buntin GD, et al. (2016) Sugarcane aphid (Hemiptera: Aphididae): A new pest on sorghum in North America. Journal of Integrated Pest Management 7: 1-13.

Colares F, Michaud JP, Bain CL, Torres JB (2015) Recruitment of aphidophagous arthropods to sorghum plants infested with Melanaphis sacchari and Schizaphis graminum (Hemiptera: Aphididae). Biological Control 90: 16-24.

da Silva M, Almeida Rocha D, Tayrane-Bezerra K, Silva D (2014) Potential population growth of Melanaphis sacchari (Zethner) reared on sugarcane and sweet sorghum. Current Agricultural Science and Technology 20: $21-25$

Domínguez RB (2011) Introducción al muestreo de plagas agrícolas. In: Anaya RS, Romero NJ (eds.) Plagas y enfermedades. Editorial Trillas, México. pp. 296-316.

Legg DE, Moon RD (1994) Bias and variability in statistical estimates. In: Pedigo LP, Buntin GD. Handbook of sampling methods for arthropods in agriculture. CRC Press, Boca Raton, FL. USA. pp. 55-69.

Naranjo SE, Flint HM (1995) Spatial distribution of adult Bemisia tabaci (Homoptera: Aleyrodidae) in cotton and development and validation of fixed-precision sampling plans for estimating population density. Environmental Entomology 24: 261-270.

Norm E, Brewer M, Seiter N, Royer T, Bowling R, Backoulou G, et al. (2017) Sugarcane aphid spatial distribution in grain sorghum fields. Southwestern Entomologist 42: 27-35.

Peña-Martínez R, Muñoz AL, Ramos G, Terrón R (2015) Listado de plantas hospedantes del complejo Melanaphis sacchari/sorghi (Hemiptera: Aphididae), registros internacionales y potenciales en México. Entomología Mexicana 2: 582-587.

Quijano-Carranza JA, Pecina-Quintero V, Bujanos-Muñiz R, Marín-Jarillo A, Yáñez-López R (2017) Guía para el manejo del pulgón amarillo del sorgo. Comité Técnico de Pulgón Amarillo del Sorgo en Guanajuato. Folleto para productores 1. Fundación Guanajuato Produce. México. 36p.

Rodríguez-del-Bosque LA, Terán AP (2015) Melanaphis sacchari (Hemiptera: Aphididae): A new sorghum insect pest in Mexico. Southwestern Entomologist 40: 433-434.

Schenck S, Lehrer AT (2000) Factors affecting the transmission and spread of sugarcane yellow leaf virus. Plant Disease 84: 1085-1088

SENASICA (2014) Pulgón amarillo Melanaphis sacchari (Zehntner). Servicio Nacional de Sanidad, Inocuidad y Calidad Agroalimentaria. Dirección General de Sanidad Vegetal-Programa Nacional de Vigilancia Epidemiológica Fitosanitaria. Ficha Técnica 43. México. 15p.

SENASICA (2017) Pulgón amarillo del sorgo. Servicio Nacional de Sanidad, Inocuidad y Calidad Agroalimentaria. https://www.gob.mx/senasica/documentos/pulgon-amarillo-del-sorgo-110905. Fecha de consulta: 18 de septiembre de 2018. 
Setokuchi O (1988) Studies on the ecology of aphids on sugarcane, I. Infestation of Melanaphis sacchari (Zehntner) (Homoptera: Aphididae). Japanese Journal of Applied Entomology and Zoology 32: 215-218.

Singh B, Padmaja P, Seetharama N (2004) Biology and management of the sugarcane aphid, Melanaphis sacchari (Zehntner) (Homoptera: Aphididae), in sorghum: A review. Crop Protection 23: 739-755.

Taylor LR (1961) Aggregation, variance and the mean. Nature 189: 732-735.

Tejeda-Reyes MA, Díaz-Nájera JF, Rodríguez-Maciel JC, Vargas-Hernández M, Solís-Aguilar JF, Ayvar-Serna S, et al. (2017) Evaluation in field of insecticides on Melanaphis sacchari (Zehntner) in sorghum. Southwestern Entomologist 42: 545-550.

Vázquez-Navarro JM, Carrillo Aguilera JC, Cisneros-Flores BA (2016) Estudio poblacional en un cultivar de sorgo forrajero infestado con pulgón amarillo del sorgo Melanaphis sacchari (Zehnter, 1897) (Hemiptera: Aphididae) en la comarca lagunera. Entomología mexicana 3: 395-400.

Villanueva RT, Brewer M, Way MO, Biles S, Sekula D, Bynum E, et al. (2014) Sugarcane aphid: a new pest of sorghum. Texas A \& M Agrilife Extension. Ento-035. Weslaco, TX, USA. http://lubbock.tamu.edu/files/2015/ 05/SCA-Management-Guide.pdf. Fecha de consulta: 18 de septiembre de 2018.

White WH, Reagan TE, Hal DG (2001) Melanaphis sacchari (Homoptera: Aphididae), a sugarcane pest new to Louisiana. Florida Entomologist 84: 435-436. 
